\title{
MRI phenotyping of underlying cerebral small vessel disease in
}

\section{mixed hemorrhage patients}

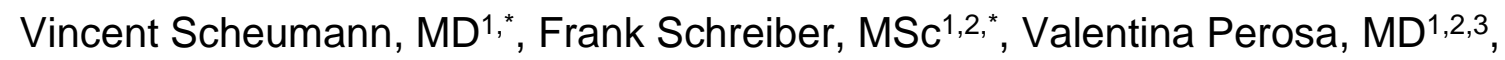
Anne Assmann, MD ${ }^{1}$, Christian Mawrin, MD ${ }^{4,6}$, Cornelia Garz ${ }^{2,5}$, Hans-Jochen Heinze, MD ${ }^{1,2,5,6}$, Michael Görtler, MD¹, Emrah Düzel, MD ${ }^{2,5,6,7}$, Stefan Vielhaber, $\mathrm{MD}^{1,2,6}$, Andreas Charidimou, $\mathrm{MD}^{8^{*}}$, Stefanie Schreiber, $\mathrm{MD}^{1,2,6^{*}}$

${ }^{*}$ These authors contributed equally to the manuscript.

${ }^{1}$ Department of Neurology, Otto-von-Guericke University, Leipziger Straße 44, 39120 Magdeburg, Germany

${ }^{2}$ German Center for Neurodegenerative Diseases (DZNE), Leipziger Straße 44, 39120 Magdeburg, Germany

3J. Philip Kistler Stroke Research Center, Massachusetts General Hospital, 175 Cambridge Street, Boston, MA 02114. USA

${ }^{4}$ Institute of Neuropathology, Otto-von-Guericke-University Magdeburg, Leipziger Straße 44, 39120 Magdeburg, Germany

${ }^{5}$ Leibniz Institute for Neurobiology (LIN), Brenneckestraße 39118 Magdeburg, Germany.

${ }^{6}$ Center for Behavioral Brain Sciences (CBBS), Universitätsplatz 239106 Magdeburg, Germany

${ }^{7}$ Institute of Cognitive Neurology and Dementia Research, Otto-von-Guericke-University Magdeburg, Leipziger Straße 44, 39120 Magdeburg, Germany

${ }^{8}$ Department of Neurology, Massachusetts General Hospital Stroke Research Center, Harvard Medical School, 55 Fruit Street, Boston, MA 02114, USA

\author{
Manuscript classification: Clinical Research Paper \\ Cover Title : \\ Title length: \\ Abstract length: \\ Manuscript length: \\ Phenotyping of underlying CSVD in mixed patients \\ 88 characters \\ 250 words \\ 3372 words \\ Submission components: 1 manuscript text, 1 figure, 2 tables \\ Reference count: \\ 40 \\ Corresponding author: \\ Stefanie Schreiber \\ Department of Neurology, \\ Otto-von-Guericke University \\ Leipziger Straße 44 \\ 39120 Magdeburg, Germany \\ Tel.: 0391-67-13431 \\ Fax: 0391-67-15233 \\ stefanie.schreiber@med.ovgu.de
}


Key words (from Medical Subject Headings database):

- intracerebral hemorrhage

- vascular dementia

- cerebral amyloid angiopathy

- cerebral small vessel diseases

- MRI

- cerebrospinal fluid

Subject Terms (from Journal Subject Terms List):

- Vascular Disease

- Cerebrovascular Disease/Stroke

- Intracranial Hemorrhage

- Imaging

- Magnetic Resonance Imaging (MRI)

- Biomarkers

- Cerebrospinal Fluid

- Cerebral Small Vessel Diseases

- Cerebral Amyloid Angiopathy

- Hypertensive Arteriopathy

- Cerebral Microbleeds

Stefanie Schreiber has full access to all of the data and takes full responsibility for the data, the analyses and interpretation, and the conduct of the research.

Vincent Scheumann and Stefanie Schreiber conducted the statistical analysis, both at Department of Neurology, Otto-von-Guericke University, Leipziger Straße 44, 39120 Magdeburg, Germany.

The study has not been supported by any sponsor and Stefanie Schreiber has the right to publish any and all data. 


\section{Disclosures}

Vincent Scheumann reports no disclosures.

Frank Schreiber reports no disclosures.

Valentina Perosa reports no disclosures.

Christian Mawrin reports no disclosures.

Anne Assmann reports no disclosures.

Cornelia Garz reports no disclosures.

Hans-Jochen Heinze reports no disclosures.

Michael Görtler reports no disclosures.

Emrah Düzel reports no disclosures.

Stefan Vielhaber reports no disclosures.

Andreas Charidimou is supported by a Bodossaki Foundation post-doctoral scholarship.

Stefanie Schreiber reports no disclosures.

Declarations of interest: none

\section{$\underline{\text { ORCID iDs }}$}

Vincent Scheumann 0000-0002-7905-0590

Frank Schreiber 0000-0002-9484-8613

Christian Mawrin 0000-0002-6677-3124

Valentina Perosa 0000-0002-3551-5237

Emrah Düzel 0000-0002-0139-5388

Andreas Charidimou 0000-0001-5891-337X

Stefanie Schreiber 0000-0003-4439-4374

\section{Email addresses:}

Vincent Scheumann vincent.scheumann@st.ovgu.de

Frank Schreiber_frank.schreiber@dzne.de

Valentina Perosa valentina.perosa@med.ovgu.de

Anne Assmann anne.assmann@med.ovgu.de

Christian Mawrin_christian.mawrin@med.ovgu.de

Cornelia Garz_cornelia.garz@med.ovgu.de

Hans-Jochen Heinze hans-jochen.heinze@med.ovgu.de

Michael Görtler@Michael.Goertler@med.ovgu.de

Emrah Düzel emrah.duezel@dzne.de

Stefan Vielhaber_stefan.vielhaber@med.ovgu.de

Andreas Charidimou acharidimou@mgh.harvard.edu

Stefanie Schreiber Stefanie.schreiber@med.ovgu.de 


\section{ABSTRACT}

OBJECTIVE: To investigate underlying cerebral small vessel disease (CSVD) in patients with mixed cerebral hemorrhages patterns and phenotype them according to the contribution of the two most common sporadic CSVD subtypes: cerebral amyloid angiopathy (CAA) vs. hypertensive arteriopathy (HA).

METHODS: Brain MRIs of patients with intracerebral hemorrhages (ICHs) and/or cerebral microbleeds (CMBs) were assessed for the full spectrum of CSVD markers using validated scales: ICHs, CMBs, cortical superficial siderosis (cSS), white matter hyperintensities, MRIvisible perivascular spaces (PVS). PVS predominance pattern was grouped as centrumsemiovale (CSO)-PVS predominance, basal-ganglia (BG)-PVS predominance, CSO-PVS and BG-PVS equality. Patients with mixed cerebral hemorrhages were classified into mixed CAA-pattern or mixed HA-pattern according to the existence of cSS and/or a CSO-PVS predominance pattern and comparisons were performed.

RESULTS: We included 110 patients with CAA (strictly lobar ICHs/CMBs), 33 with HA (strictly deep ICHs/CMBs) and 97 with mixed lobar/deep ICHs/CMBs. Mixed patients were more similar to HA with respect to their MRI-CSVD markers, vascular risk profile and cerebrospinal fluid (CSF) measures. In the mixed patients, $33(34 \%)$ had cSS, a CSO-PVS predominance pattern, or both, and were defined as mixed CAA-pattern cases. The mixed CAA-pattern patients were more alike CAA patients regarding their MRI-CSVD markers, CSF and genetic profile.

CONCLUSION: Our findings suggest that the heterogeneous group of patients with mixed cerebral hemorrhages distribution can be further phenotyped according to the predominant underlying CSVD. cSS presence and a CSO-PVS predominance pattern could serve as strongly suggestive markers of a contribution from CAA among patients with mixed hemorrhages. 


\section{ABBREVIATIONS}

$A \beta$

AD

BG

ApoE

CAA

CHARTS

CMB

CMB-R

CSF

CSO

cSS

CSVD

PVS

FLAIR

GRE

HA

HDL

ICH

MARS

ttau

ptau

STRIVE STandards for Reportlng Vascular Changes on nEuroimaging

SWI

TE

TR

WMH

Amyloid- $\beta$

Alzheimer's disease

Basal ganglia

Apolipoprotein E

Cerebral amyloid angiopathy

Cerebral Haemorrhage Anatomical RaTing instrument

Cerebral microbleed

Cerebral microbleed ratio

Cerebrospinal fluid

Centrum semiovale

Cortical superficial siderosis

Cerebral small vessel disease

MRI-visible perivascular spaces

Fluid-attenuated inversion recovery

Gradient-recalled echo

Hypertensive arteriopathy

High-density lipoprotein

Spontaneous intracerebral hemorrhage

Microbleed Anatomical Rating Scale

Total tau

Phosphorylated tau

Susceptibility-weighted imaging

Echo time

Repetition time

White matter hyperintensities 


\section{INTRODUCTION}

2 The two common subtypes of sporadic cerebral small vessel disease (CSVD) are cerebral

3 amyloid angiopathy (CAA) and hypertensive arteriopathy (HA)[1]. The pattern of associated

4 brain hemorrhages reflects a mirror distribution: CAA is associated with strictly lobar and HA

5 with deep gray matter hemorrhages, though they can also occur in lobar regions at advanced

6 disease[2-4].

7 The modified Boston Criteria provide high diagnostic certainty for CAA when blood-sensitive

8 magnetic resonance imaging (MRI) sequences demonstrate multiple lobar hemorrhages[5],

9 including cortical superficial siderosis (cSS) (a highly specific CAA signature)[6]. A 10 predominance pattern of MRI-visible perivascular spaces (PVS) in the centrum semiovale 11 (CSO) vs. basal ganglia (BG) is further strongly associated with CAA, but not $\mathrm{HA}[7]$.

12 In clinical practice, up to $40 \%$ of all CSVD patients show a pattern of mixed lobar and deep 13 hemorrhages[8,9]. According to available clinical-radiologic criteria, these patients cannot be 14 classified as having CAA or HA, leaving their stratification and therapeutic advice an open 15 question. Two possibilities exist in these patients: (a) the co-occurrence of both CAA and HA; 16 (b) the presence of advanced $\mathrm{HA}[8,10]$.

17 We hypothesize, that applying MRI markers that are specific for CAA (cSS, CSO-PVS 18 predominance) might allow a more precise and clinically relevant classification of CSVD 19 patients with mixed hemorrhages into (a) a mixed "CAA-pattern"; and (b) a mixed "HA20 pattern". We aimed to demonstrate the validity of this classification, by showing that the 21 mixed CAA-pattern group should be more similar to CAA patients and the mixed HA-pattern 22 group to HA patients with regard to their imaging and clinical characteristics. 


\section{MATERIALS AND METHODS}

\section{$24 \quad 2.1 \quad$ Study population}

25 We designed a retrospective cross-sectional study and screened our neuroimaging database 26 for cerebral MRI (cMRI) scans with blood-sensitive sequences conducted for diagnostic 27 work-up at the University Clinic Magdeburg between 10/21/2003 and 07/31/2020. Patients 28 were included in the study if they had spontaneous $\mathrm{ICH}(\mathrm{s})$ and/or $\mathrm{CMBs}$ on cMRI; $\mathrm{n}=262$ subjects were identified. We excluded $n=22(13 \%)$ because of having just a single lobar CMB histopathological correlation studies) has low specificity for underlying CAA[11], or because of poor scan quality $(n=6)$ (Supplemental Figure I). There was a remainder of $n=240$ patients presenting the final study cohort with the following clinical diagnoses: $n=117(49 \%)$ had $\mathrm{ICH}, \mathrm{n}=50(21 \%)$ ischemic (i.e. lacunar) stroke or transient ischemic attack, $\mathrm{n}=43(18 \%)$ dementia (i.e. Alzheimer's disease [AD] or vascular dementia), $n=8(3 \%)$ epileptic seizures, $n=8 \quad(3 \%)$ subarachnoid hemorrhage, $n=4 \quad(2 \%)$ CAA-related inflammation, $n=5 \quad(3 \%)$ Parkinson's disease, $n=1(1 \%)$ multiple sclerosis, $n=2(1 \%)$ renal encephalopathia, and $n=2$ $(1 \%)$ in whom cerebral metastases were initially suspected, but excluded through MRI.

According to the locations of ICHs and CMBs, patients were divided into three groups: strictly lobar ICHs and/or CMBs (probable CAA according to the modified Boston criteria), strictly deep $\mathrm{ICHs}$ and/or CMBs (HA), lobar and deep ICHs and/or CMBs in any combination (mixed). Cerebellar ICHs and/or CMBs were allowed to occur in all three groups, but did not count in the classification. Mixed patients were further split into a mixed CAA-pattern group and a mixed HA-pattern group. Split was based on the existence of cSS or CSO-PVS predominance pattern, which defined the mixed CAA-pattern group, and was absent in the mixed HA-pattern group[6,7,12,13]. Subtyping/split of mixed patients was not based on the presence or number of hemorrhages (ICHs, CMBs).

In addition, the cohort was characterized with regard to its demographic (age and sex), clinical (existence of arterial hypertension, diabetes mellitus, hyperlipidemia, obesity), cerebrospinal fluid (CSF, see below) and apolipoprotein $E$ (ApoE) data. 
2.2 Standard Protocol Approvals, Registrations, and Patient Consents

52 This retrospective study was approved by the local ethics committee (No. 28/16).

\subsection{MRI acquisition}

MRI was performed using a $1.5 \mathrm{~T}$ (Siemens Healthineers; $n=126[53 \%]$ of the patients) and $3 T$ Scanner (Philips Medical Systems; $n=114[48 \%]$ ), including T2*-gradient-recalled echo (GRE) (slice thickness: 3 to $4 \mathrm{~mm}$, repetition time [TR]: 500 to 1.000 milliseconds, echo time [TE]: 11-13 milliseconds) or susceptibility-weighted imaging (SWI) (slice thickness: 1 to $2 \mathrm{~mm}$, TR: 20 milliseconds, TE: 20-40 milliseconds), fluid-attenuated inversion recovery (FLAIR) (slice thickness: 4 to $5 \mathrm{~mm}$, TR: 6.000 to 11.000 milliseconds, TE: 90 to 140 milliseconds), and T2 sequences (slice thickness: 3 to $4 \mathrm{~mm}$, TR: 3.000 to 1.000 milliseconds, TE: 80 to 10 milliseconds).

\section{$2.4 \quad$ MRI analysis}

MRI analysis of all patients was performed in a semiquantitative manner according to the Standards for Reporting Vascular Changes on Neuroimaging (STRIVE)[14] by a trained investigator (VS), blinded to all demographic, clinical, CSF and genetic information. The images were evaluated using specific software (Mango for 1.5T dicom images, Osirix for 3T nii images) and established methods and scales (see below). With the exception of PVS (see below), per patient all available MRI slices were analyzed, respectively. Intra-rater reliability based on a sample of 20 randomly chosen cases and inter-rater reliability based on a sample of 11 randomly chosen cases (by a second independent and blinded rater (VP)) was excellent for all investigated variables (intra-class correlation coefficient (ICC) $>0.86$ and $>0.81$, respectively).

CMBs were defined as small (diameter $2-5 \mathrm{~mm}$, maximum up to $10 \mathrm{~mm}$ ), round or oval in axial T2*-GRE ( $n=235) / S W I(n=5)$ hypointense lesions, not visible in FLAIR, T1-, or T2-weighted sequences. They were categorized into lobar (frontal, temporal, parietal, occipital, insula), deep (BG, thalamus, internal capsule, external capsule, corpus callosum, deep and periventricular white matter, brainstem), and infratentorial (cerebellum) CMBs applying the 
Microbleed Anatomical Rating Scale (MARS)[14-16]. The total CMB count was calculated for

79 the whole-brain and for each anatomical region separately (lobar, deep, infratentorial). 80 Additionally, a CMB ratio (CMB-R) was created by dividing the number of lobar CMBs 81 through the number of deep CMBs[17].

$82 \mathrm{ICH}$ s (diameter $>10 \mathrm{~mm}$ ) were classified using axial T2*-GRE sequences $(n=235)$ or SWI $83(n=5)$ according to the Cerebral Haemorrhage Anatomical RaTing instrument (CHARTS) as 84 lobar (frontal, parietal, temporal, occipital, insular), deep (BG, thalamus, brainstem) and 85 infratentorial ICHs (cerebellum)[18]. ICHs were summed up for the whole brain and for each region, as described for CMBs (see above). Anatomical location was determined according 87 to the largest size/diameter and epicenter of the $\mathrm{ICH}[14,18,19]$.

88 cSS was defined as a homogeneous T2*-GRE/SWI hypointensity found in the superficial 89 cortex layers and subarachnoid space corresponding to hemosiderin deposition[13]. cSS 90 was evaluated qualitatively on axial sequences using T2*-GRE $(n=235)$ or SWI $(n=5)$ and 91 categorized as present/absent and classified as either focal (restricted to $\leq 3$ sulci) or 92 disseminated ( $\geq 4$ sulci), in line with the modified Boston Criteria[6].

93 White matter hyperintensities of presumed vascular origin (WMHs) were divided into four 94 recently defined patterns (present or absent): (1) multiple subcortical spots, (2) peri-BG 95 ("around BG") WMHs, (3) anterior subcortical pattern, and (4) posterior subcortical 96 pattern[20]. We thereby took account of axial FLAIR images $(n=202)$, or, if absent, coronary $97 \quad$ FLAIR images $(n=38)$.

98 MRI-visible PVS are fluid-filled spaces around small vessels with a maximum diameter of $993 \mathrm{~mm}$ and a CSF-like signal behavior on FLAIR and T2-weighted images[21]. The severity of PVS was counted separately in the CSO (above the lateral ventricle/corpus callosum) and the BG. CSO and BG PVS were graded using axial T2- $(n=174)$, and/or sagittal T2-weighted $(n=66)$ scans[22]. CSO-PVS analysis took place in planes superior to the lateral ventricles/corpus callosum. For the BG the caudate nucleus, internal capsule, thalamus, lentiform nucleus, external/extreme capsules, and insular cortex were taken into account. At least 3 slices per subject were reviewed for the number of CSO- and BG-PVS, respectively, 
106 taking account of both hemispheres, but counting the side (left or right) with the highest PVS

number only. CSO- and BG-PVS were classified separately as either mild to moderate $(<20$ PVS) or frequent to severe (>20 PVS). In addition, each individual patient was assigned to one of the following three categories comparing the degree of CSO-PVS and BG-PVS: CSOPVS predominance (higher degree of CSO-PVS: CSO-PVS > BG-PVS), BG-PVS predominance (higher degree of BG-PVS; BG-PVS > CSO-PVS), or equal degree of PVS in the CSO and BG (CSO-PVS = BG-PVS) [7].

\subsubsection{CSF measures}

Within 20 minutes of lumbar puncture, CSF samples were centrifuged at $4^{\circ} \mathrm{C}$, aliquoted and stored at $-80^{\circ} \mathrm{C}$ until analysis. CSF biomarkers were measured with commercially available ELISA (for $A \beta$ 1-40: Innotest $\beta$-Amyloid(1-40); for $A \beta$ 1-42: Innotest $\beta$-Amyloid(1-42); for total tau (ttau): Innotest hTauAg; for phosphorylated tau (ptau): Innotest p-Tau; Fujirebio, Ghent, Belgium), following the instructions provided by the manufacturer. Locally established thresholds were $485 \mathrm{pg} / \mathrm{ml}$ for $A \beta$ 1-42, 350pg/ml for ttau and $70 \mathrm{pg} / \mathrm{ml}$ for ptau[23]. In addition, we also determined the $A \beta$ 1-42/A $\beta-1-40$ ratio - which is commonly used for $A \beta$ pathology detection - to normalize CSF A 1-42 in terms of a better control for fluctuations in total CSF amyloid levels[24-27].

\section{$2.5 \quad$ Statistical analysis}

Shapiro-Wilk test was used to assess Gaussian distribution of the data. For group comparisons between CAA, HA and mixed, Kruskal-Wallis one-way analysis of variance (ANOVA) with post hoc pairwise Mann-Whitney U testing or ANOVA with Bonferroni post hoc testing was conducted. For group comparisons between mixed CAA-pattern and mixed HApattern, a Mann-Whitney $U$ test or an independent-samples t-test was used. Bivariate variables were analyzed using logistic regression analysis. Age and sex were always considered as covariates. Significance levels for group comparisons were determined after Bonferroni adjustment for 17 studied imaging markers as $p \leq 0.05 / 17=2.9 \cdot 10^{-3}$. Analyses 132 were performed using the IBM SPSS Statistics 24.0 software. 


\subsection{Data availability}

1

2134 Anonymized grouped data will be shared by request from a qualified investigator.

3

4

5

6

7

8

9

10

11

12

13

14

15

16

17

18

19

20

21

22

23

24

25

26

27

28

29

30

31

32

33

34

35

36

37

38

39

40

41

42

43

44

45

46

47

48

49

50

51

52

53

54

55

56

57

58

59

60

61

62

63

64

65

$11 / 26$ 


\section{RESULTS} given in Table 2. (Figure 1E-H).

MRI markers and demographics, clinical, CSF and genetic data for the whole cohort, comprising CAA, HA and mixed patients, are given in Table 1. Our final cohort included 110(46\%) CAA patients, 33(14\%) HA and 97(40\%) patients classified as mixed. A total of $\mathrm{n}=117(49 \%)$ patients have suffered from ICH; $80(33 \%)$ had lobar $\mathrm{ICH}, 33(14 \%)$ deep and 4 (1\%) cerebellar $\mathrm{ICH}$. Total $\mathrm{ICH}$ prevalence did not differ between CAA, HA and mixed cases (Supplemental Table I).

CAA compared to HA and mixed patients was associated with cSS, multiple subcortical WMH spots, severe CSO-PVS, a CSO-PVS predominance pattern, and less frequent periBG WMHs and severe BG-PVS. In addition, CAA compared to the mixed group had less cerebellar CMBs and a lower frequency of anterior subcortical WMHs (trend). HA compared to mixed patients had more deep ICHs, less deep and cerebellar CMBs and lower prevalence of severe CSO-PVS (Table 1). Patients with CAA tended to be older than HA or mixed cases. In CAA compared to mixed patients, prevalence was lower for arterial hypertension (trend) and diabetes mellitus (trend); CAA patients were further less obese and had higher HDL levels (trend). CAA compared to HA or mixed was related to a lower A 1 42/A $\beta$ 1-40 ratio and a higher prevalence of $A \beta$ 1-42-positivity (trend) (Table 1).

\subsection{Mixed CAA-pattern vs. mixed HA-pattern}

MRI markers and demographics, clinical, CSF and genetic data for the patients with mixed cerebral hemorrhages, comparing the mixed CAA-pattern and mixed HA-pattern groups, are

Of the 97 patients with mixed cerebral hemorrhages, $n=33(34 \%)$ patients had cSS $(n=5(5 \%))$, a CSO-PVS predominance pattern $(n=17(18 \%))$ or both (i.e. cSS and a CSOPVS predominance pattern $(n=11(11 \%)))$ and were therefore classified as mixed CAApattern (Figure 1A-D). The remainder of $n=64(66 \%)$ was classified as mixed HA-pattern 
161 Mixed CAA-pattern compared to mixed HA-pattern showed trends towards more lobar 9

CMBs, a higher CMB-R, more lobar and less deep ICHs, and significantly less frequently peri-BG WMHs. Mixed CAA-pattern patients tended to be more often A 3 1-42 positive, along with trends towards a lower $A \beta$ 1-42/A -1-40 ratio and more ApoE\&4-carriers than mixed HApattern cases (Table 2).

\subsection{Mixed CAA-pattern vs. CAA and mixed HA-pattern vs. HA}

Comparison of MRI markers and demographics, clinical, CSF and genetic data between mixed CAA-pattern vs. CAA and mixed HA-pattern vs. HA is given in Supplemental Table II and Supplemental Table III. Patients with mixed CAA-pattern compared to CAA were younger, more often female (trend), had lower HDL and a higher $A \beta$ 1-42/A $\beta-1-40$ ratio (trend). Mixed HA-pattern compared to HA had more commonly CMBs, less deep ICHs, 172 trends towards higher frequencies of posterior subcortical $\mathrm{WMH}$ patches and arterial 173 hypertension. 
In this cross-sectional study we provide proof-of-concept evidence for MRI phenotyping of underlying CSVD subtypes in mixed hemorrhage patients. We tested the prespecified hypothesis that mixed hemorrhage patients could either have a (a) co-occurrence of CAA and HA, or (b) advanced HA affecting deep and lobar regions, and developed a simple MRI approach to potentially discriminate the two. Our results indicate that mixed hemorrhage patients with a mixed CAA-pattern (34\% of mixed patients) could be identified through the presence of cSS and/or CSO-PVS predominance. Mixed hemorrhage patients without cSS and/or CSO-PVS predominance seem to better fit into a category of a mixed HA-pattern (66\%).

184 We confirmed that, at a group-level, a mixed cerebral hemorrhage pattern is associated with 185 a higher vascular risk profile when compared to CAA. As a whole, the mixed group showed 186 more similarities with HA patients with regard to arterial hypertension, diabetes mellitus, 187 obesity and dyslipidemia prevalence. These results replicate findings of a recent study that 188 showed that patients with a mixed hemorrhage pattern presented a clinical phenotype 189 sharing more similarities with patients with a deep hemorrhage than with those with a lobar 190 hemorrhage pattern[8]. Also with regard to their MRI and CSF characteristics, there were far 191 more similarities between mixed and HA patients than between mixed and CAA patients.

192 Interestingly, up to $34 \%$ of the mixed patients displayed a mixed CAA-pattern, in terms of 193 prespecified neuroimaging features that are highly specific for CAA, i.e. cSS presence and 194 CSO-PVS predominance. Hence, the mixed cerebral hemorrhage patient group is more 195 heterogeneous than appreciated, and not all mixed patients have an advanced HA-driven CSVD process. Recent studies support this hypothesis in that the mixed hemorrhage group also shares some similarities with $\mathrm{CAA}$, such as increased risk for $\mathrm{ICH}$ recurrence or higher amyloid load[8,28]. The two putative CAA MRI markers used in our approach seem thereby to provide a strong indication of at least concomitant advanced underlying CAA cases within this mixed group. Accordingly, mixed CAA-pattern patients had a higher prevalence of lobar 
201 hemorrhages (ICHs, CMBs) and a higher CMB-R, confirming that our subtyping method

1

results in a predominant lobar hemorrhage pattern quite characteristic for CAA. Likewise, mixed CAA-pattern patients had less frequently peri-BG WMHs, recently proposed to be an imaging marker for $\mathrm{HA}[20]$. Furthermore, mixed CAA-pattern was related to CSF A 3 1-42 positivity and ApoE\&4 carrier status, mirroring the association between CAA and low CSF A $\beta$ 1-42 levels and ApoEє4 positivity[24,26,29-34]. Both low CSF A $\beta$ 1-42 concentration and low CSF $A \beta$ 1-42/A 1 1-40 ratio (which was found in the CAA-mixed pattern as well) indicate the deposition of the $A \beta$ 1-42 isoform in the brain parenchyma and, to a lesser extent, in the cerebral vasculature. Positive CSF $A \beta$ 1-42 biomarker status and a low CSF A $\beta$ 1-42/A $\beta$ 140 ratio both, together with CSF ptau elevation, are commonly used to determine $A D$ pathology comprising parenchymal $A \beta[31,32]$. The fact, that just a minority of the mixed CAA-pattern group had AD (3\%) and that CSF ptau was unaltered, points towards vascular $A \beta$ 1-42 deposition to be the (dominant) driver of the CSF $A \beta$ findings in the CAA-mixed pattern patient group.

Compared to mixed CAA-pattern, mixed HA-pattern had more often peri-BG WMHs and less often signature findings determining CAA (e.g. less lobar $\mathrm{CMBs} / \mathrm{ICH}$, a lower $C M B-\mathrm{R}$, a lower prevalence of CSF A $\beta$ 1-42 positivity). Those findings deem mixed HA-pattern to reflect HA. Interestingly, when comparing mixed HA-pattern against HA, in mixed HA-pattern patients HA seemed to be even more severe as indicated by a higher load of deep CMBs, more extended WMHs and a higher prevalence of arterial hypertension. The latter results let us speculate that mixed HA-pattern fit the proposed category (b) of advanced HA affecting deep and lobar regions. Supporting our classification, a 56-year-old male patient classified as mixed MRI HA-pattern underwent brain biopsy to elucidate the underlying pathology of rapidly progressive cognitive decline together with $\mathrm{WMH}$. MRI rating displayed mixed CMBs, equally severe PVS in BG and CSO, and WMH with multiple, subcortical spots (Supplemental Figure II). Neuropathology examination demonstrated no CAA (Vonsattel grade 0) [35] but severe arteriolosclerosis, i.e. HA. Further details about the patient's clinical and biomarker data are displayed in Supplemental Table IV. 
229 The strength of our study is the comparatively high number of mixed hemorrhage patients,

1 compared to previous studies which typically included significantly less mixed cases $[8,9,17,33]$. This high occurrence rate of such mixed cases in a hospital-based population points to the clinical relevance of our study, seeking for a better understanding of the presumed underlying CSVD pathology in this particular patient population. In addition, we assessed the full spectrum of MRI CSVD markers using standardized and validated scores to test our prespecified hypothesis. We provided compelling evidence that cohorts of mixed hemorrhages are heterogeneous with regards to the underlying CSVD and provided an actionable strategy to further subgroup them based on key MRI markers. The use of MRIs from everyday clinical practice makes our findings easily translated for clinical practice. Our results could be of significance for both, further research and clinical practice, and additional prospective studies with adequate sample sizes are needed to validate our findings. Potentially, our proposed classification, i.e. mixed cerebral hemorrhages together with CSS and/or PVS-CSO predominance, could become a useful addition to the modified Boston criteria to find CAA cases within the mixed group. This approach also highlights the growing significance of non-hemorrhagic markers for CSVD subtype classification[21,36]. Thus, hemorrhagic and non-hemorrhagic markers together will prospectively help to aid in a more subtle classification of CAA and non-CAA CSVD patients. One may consider that mixed CAA-pattern cases might behave, to a certain extent, more similar to CAA patients: they could have a higher risk of recurrent $\mathrm{ICHs}$, especially under oral anticoagulation or intravenous thrombolysis, and of cognitive decline, particularly in contrast to $\mathrm{HA}$ patients[2,34,37]. These aspects make the identification of mixed CAA-pattern cases within the mixed group a clinically highly relevant goal; they thus need to be considered in the future focusing on longitudinal studies.

Our study has some limitations. First, patients were selected retrospectively according to the existence of $\mathrm{ICHs}$ and/or $\mathrm{CMBs}$ within MRIs that were performed as a part of routine diagnostic work-up. As a result, the scanning protocol was not completely harmonized and different imaging parameters and sequences might affect CSVD markers assessments. 
257 Likewise, there were several missing data especially for CSF and genotyping. This is

1

2 3 4

6 8 9 10

explained by the fact, that this diagnostic is not part of the routine work-up for ICH or ischemic stroke/transient ischemic attack presented by $2 / 3$ of the patients of our cohort. The time of recruitment (from 2003 to 2020) is very long which lead to a changing MRI acquisition - using 1.5T as well as 3T MRI. Furthermore, the study population is heterogeneous and in a small part of the population the finding of $\mathrm{CMB}$ on MRI could be incidental. Selection bias could be another limitation in that diagnostic MRI in the clinic is usually performed in more stable patients. Lastly, in the mixed patients there was a relationship between cSS and ICH presence (data not shown), which has already been well established in former studies[38,39]. CSS presence could thus be considered a proxy for higher ICH prevalence in the mixed CAA group. In our cohort, ICH prevalence nevertheless did not significantly differ

268 between mixed CAA and mixed HA (61\% vs. $41 \%, \mathrm{p}=0.06)$. We are thus convinced, that ICH 269 presence has not introduced a bias towards assigning the mixed patients to the mixed CAA 270 group. 


\section{CONCLUSIONS}

6 7 8

In conclusion, our findings indicate that patients with mixed cerebral hemorrhages are highly prevalent amongst CSVD patients with and without ICHs. This group as a whole has a vascular risk profile, which is similar to that of HA patients. Our study provided novel evidence that that one third of patients with mixed cerebral hemorrhages present a more CAA-pattern phenotype, easily characterized on clinical MRI by the assessment of two key imaging markers - cSS and CSO-PVS predominance. Our approach requires external validation in larger patient cohorts. Further work will need to explore mixed cerebral hemorrhage subgroup and phenotypes with regards to risk profile in terms of $\mathrm{ICH}$ recurrence and cognitive decline. Neuropathological studies are finally warranted to confirm and refine 281 the contribution of suspected CAA within the mixed CAA-pattern phenotype. 


\begin{tabular}{|c|c|c|c|}
\hline Name & Location & Role & Contribution \\
\hline Vincent Scheumann, MD & $\begin{array}{l}\text { Otto-von-Guericke University, } \\
\text { Magdeburg, Germany }\end{array}$ & Author & $\begin{array}{l}\text { Design and conceptualized study; analyzed the data; } \\
\text { drafted the manuscript for intellectual content }\end{array}$ \\
\hline Frank Schreiber, MSc & $\begin{array}{l}\text { Otto-von-Guericke University, } \\
\text { Magdeburg, Germany }\end{array}$ & Author & $\begin{array}{l}\text { Acquired, administrated and analyzed the data, revised } \\
\text { the manuscript for intellectual content }\end{array}$ \\
\hline Valentina Perosa, MD & $\begin{array}{l}\text { Otto-von-Guericke University, } \\
\text { Magdeburg, Germany }\end{array}$ & Author & Revised the manuscript for intellectual content \\
\hline Anne Assmann, MD & $\begin{array}{l}\text { Otto-von-Guericke University, } \\
\text { Magdeburg, Germany }\end{array}$ & Author & Revised the manuscript for intellectual content \\
\hline Christian Mawrin, MD & $\begin{array}{l}\text { Otto-von-Guericke University, } \\
\text { Magdeburg, Germany }\end{array}$ & Author & Acquired, administrated and analyzed the data \\
\hline Cornelia Garz & $\begin{array}{l}\text { Otto-von-Guericke University, } \\
\text { Magdeburg, Germany }\end{array}$ & Author & Acquired, administrated and analyzed the data \\
\hline Hans-Jochen Heinze, MD & $\begin{array}{l}\text { Otto-von-Guericke University, } \\
\text { Magdeburg, Germany }\end{array}$ & Author & Revised the manuscript for intellectual content \\
\hline Michael Görtler, MD & $\begin{array}{l}\text { Otto-von-Guericke University, } \\
\text { Magdeburg, Germany }\end{array}$ & Author & Revised the manuscript for intellectual content \\
\hline Emrah Düzel, MD & $\begin{array}{l}\text { Otto-von-Guericke University, } \\
\text { Magdeburg, Germany }\end{array}$ & Author & Revised the manuscript for intellectual content \\
\hline Stefan Vielhaber, MD & $\begin{array}{l}\text { Otto-von-Guericke University, } \\
\text { Magdeburg, Germany }\end{array}$ & Author & Revised the manuscript for intellectual content \\
\hline Andreas Charidimou, MD & $\begin{array}{ll}\text { Massachusetts } & \text { General } \\
\text { Hospital, Boston, USA } & \end{array}$ & Author & $\begin{array}{l}\text { Conceptualized study; interpreted the data; revised the } \\
\text { manuscript for intellectual content }\end{array}$ \\
\hline Stefanie Schreiber, MD & $\begin{array}{l}\text { Otto-von-Guericke University, } \\
\text { Magdeburg, Germany }\end{array}$ & Author & $\begin{array}{l}\text { Design and conceptualized study; analyzed the data; } \\
\text { drafted the manuscript for intellectual content }\end{array}$ \\
\hline
\end{tabular}




\section{References}

[1] A. Charidimou, L. Pantoni, S. Love, The concept of sporadic cerebral small vessel disease: A road map on key definitions and current concepts, Int J Stroke. 11 (2016) 6-18. https://doi.org/10.1177/1747493015607485.

[2] L. Pantoni, Cerebral small vessel disease: from pathogenesis and clinical characteristics to therapeutic challenges, Lancet Neurol. 9 (2010) 689-701. https://doi.org/10.1016/S1474-4422(10)70104-6.

[3] A. Charidimou, S. Martinez-Ramirez, Y.D. Reijmer, J. Oliveira-Filho, A. Lauer, D. Roongpiboonsopit, M. Frosch, A. Vashkevich, A. Ayres, J. Rosand, M.E. Gurol, S.M. Greenberg, A. Viswanathan, Total Magnetic Resonance Imaging Burden of Small Vessel Disease in Cerebral Amyloid Angiopathy: An Imaging-Pathologic Study of Concept Validation, JAMA $\quad$ Neurol. $\quad 73 \quad$ (2016) 994-1001. https://doi.org/10.1001/jamaneurol.2016.0832.

[4] G.A. Dierksen, M.E. Skehan, M.A. Khan, J. Jeng, R.N.K. Nandigam, J.A. Becker, A. Kumar, K.L. Neal, R.A. Betensky, M.P. Frosch, J. Rosand, K.A. Johnson, A. Viswanathan, D.H. Salat, S.M. Greenberg, Spatial relation between microbleeds and amyloid deposits in amyloid angiopathy, Ann. Neurol. 68 (2010) 545-548. https://doi.org/10.1002/ana.22099.

[5] K.A. Knudsen, J. Rosand, D. Karluk, S.M. Greenberg, Clinical diagnosis of cerebral amyloid angiopathy: validation of the Boston criteria, Neurology. 56 (2001) 537-539.

[6] J. Linn, A. Halpin, P. Demaerel, J. Ruhland, A.D. Giese, M. Dichgans, M.A. van Buchem, H. Bruckmann, S.M. Greenberg, Prevalence of superficial siderosis in patients with cerebral amyloid angiopathy, Neurology. $74 \quad$ (2010) 1346-1350. https://doi.org/10.1212/WNL.0b013e3181dad605.

[7] A. Charidimou, G. Boulouis, M. Pasi, E. Auriel, E.S. van Etten, K. Haley, A. Ayres, K.M. Schwab, S. Martinez-Ramirez, J.N. Goldstein, J. Rosand, A. Viswanathan, S.M. Greenberg, M.E. Gurol, MRI-visible perivascular spaces in cerebral amyloid angiopathy and hypertensive arteriopathy, Neurology. $88 \quad$ (2017) $1157-1164$. https://doi.org/10.1212/WNL.0000000000003746.

[8] M. Pasi, A. Charidimou, G. Boulouis, E. Auriel, A. Ayres, K.M. Schwab, J.N. Goldstein, J. Rosand, A. Viswanathan, L. Pantoni, S.M. Greenberg, M.E. Gurol, Mixedlocation cerebral hemorrhage/microbleeds: Underlying microangiopathy and recurrence risk, Neurology. 90 (2018) e119-e126. https://doi.org/10.1212/WNL.0000000000004797.

[9] E.E. Smith, K.R.N. Nandigam, Y.-W. Chen, J. Jeng, D. Salat, A. Halpin, M. Frosch, L. Wendell, L. Fazen, J. Rosand, A. Viswanathan, S.M. Greenberg, MRI markers of small vessel disease in lobar and deep hemispheric intracerebral hemorrhage, Stroke. 41 (2010) 1933-1938. https://doi.org/10.1161/STROKEAHA.110.579078.

[10] S. Martinez-Ramirez, S.M. Greenberg, A. Viswanathan, Cerebral microbleeds: overview and implications in cognitive impairment, Alzheimers Res Ther. 6 (2014) 33. https://doi.org/10.1186/alzrt263.

[11] S.M. Greenberg, A. Charidimou, Diagnosis of Cerebral Amyloid Angiopathy: Evolution of the Boston Criteria, Stroke. 49 (2018) 491-497. https://doi.org/10.1161/STROKEAHA.117.016990.

[12] A. Charidimou, R.H. Jäger, Z. Fox, A. Peeters, Y. Vandermeeren, P. Laloux, J.-C. Baron, D.J. Werring, Prevalence and mechanisms of cortical superficial siderosis in cerebral $\begin{array}{lllll}\text { amyloid } \quad \text { angiopathy, } & \text { Neurology. } & \text { 626-632. }\end{array}$ https://doi.org/10.1212/WNL.0b013e3182a08f2c.

[13] A. Charidimou, J. Linn, M.W. Vernooij, C. Opherk, S. Akoudad, J.-C. Baron, S.M. Greenberg, H.R. Jäger, D.J. Werring, Cortical superficial siderosis: detection and clinical significance in cerebral amyloid angiopathy and related conditions, Brain. 138 (2015) 21262139. https://doi.org/10.1093/brain/awv162.

[14] J.M. Wardlaw, E.E. Smith, G.J. Biessels, C. Cordonnier, F. Fazekas, R. Frayne, R.I. Lindley, J.T. O'Brien, F. Barkhof, O.R. Benavente, S.E. Black, C. Brayne, M. Breteler, H. Chabriat, C. Decarli, F.-E. de Leeuw, F. Doubal, M. Duering, N.C. Fox, S. Greenberg, V. Hachinski, I. Kilimann, V. Mok, R. van Oostenbrugge, L. Pantoni, O. Speck, B.C.M. Stephan, 
S. Teipel, A. Viswanathan, D. Werring, C. Chen, C. Smith, M. van Buchem, B. Norrving, P.B. Gorelick, M. Dichgans, STandards for Reportlng Vascular changes on nEuroimaging (STRIVE v1), Neuroimaging standards for research into small vessel disease and its contribution to ageing and neurodegeneration, Lancet Neurol. 12 (2013) 822-838. https://doi.org/10.1016/S1474-4422(13)70124-8.

[15] S.M. Gregoire, U.J. Chaudhary, M.M. Brown, T.A. Yousry, C. Kallis, H.R. Jäger, D.J. Werring, The Microbleed Anatomical Rating Scale (MARS): reliability of a tool to map brain microbleeds, Neurology. 73 (2009) 1759-1766. https://doi.org/10.1212/WNL.0b013e3181c34a7d.

[16] S.M. Greenberg, M.W. Vernooij, C. Cordonnier, A. Viswanathan, R. Al-Shahi Salman, S. Warach, L.J. Launer, M.A. Van Buchem, M.M. Breteler, Microbleed Study Group, Cerebral microbleeds: a guide to detection and interpretation, Lancet Neurol. 8 (2009) 165-174. https://doi.org/10.1016/S1474-4422(09)70013-4.

[17] H.-H. Tsai, L.-K. Tsai, Y.-F. Chen, S.-C. Tang, B.-C. Lee, R.-F. Yen, J.-S. Jeng, Correlation of Cerebral Microbleed Distribution to Amyloid Burden in Patients with Primary Intracerebral Hemorrhage, Sci Rep. 7 (2017) 44715. https://doi.org/10.1038/srep44715.

[19] F. Macellari, M. Paciaroni, G. Agnelli, V. Caso, Neuroimaging in intracerebral hemorrhage, Stroke. 45 (2014) 903-908. https://doi.org/10.1161/STROKEAHA.113.003701.

[20] A. Charidimou, G. Boulouis, K. Haley, E. Auriel, E.S. van Etten, P. Fotiadis, Y. Reijmer, A. Ayres, A. Vashkevich, Z.Y. Dipucchio, K.M. Schwab, S. Martinez-Ramirez, J. Rosand, A. Viswanathan, S.M. Greenberg, M.E. Gurol, White matter hyperintensity patterns in cerebral amyloid angiopathy and hypertensive arteriopathy, Neurology. 86 (2016) 505511. https://doi.org/10.1212/WNL.0000000000002362.

[21] S. Martinez-Ramirez, O.M. Pontes-Neto, A.P. Dumas, E. Auriel, A. Halpin, M. Quimby, M.E. Gurol, S.M. Greenberg, A. Viswanathan, Topography of dilated perivascular spaces in subjects from a memory clinic cohort, Neurology. 80 (2013) 1551-1556. https://doi.org/10.1212/WNL.0b013e31828f1876.

[22] F.N. Doubal, A.M.J. MacLullich, K.J. Ferguson, M.S. Dennis, J.M. Wardlaw, Enlarged perivascular spaces on MRI are a feature of cerebral small vessel disease, Stroke. 41 (2010) 450-454. https://doi.org/10.1161/STROKEAHA.109.564914.

[23] P. Körtvelyessy, H.J. Heinze, J. Prudlo, D. Bittner, CSF Biomarkers of Neurodegeneration in Progressive Non-fluent Aphasia and Other Forms of Frontotemporal Dementia: Clues for Pathomechanisms?, Front Neurol. 9 (2018) 504. https://doi.org/10.3389/fneur.2018.00504.

[24] J. Wiltfang, H. Esselmann, M. Bibl, M. Hüll, H. Hampel, H. Kessler, L. Frölich, J. Schröder, O. Peters, F. Jessen, C. Luckhaus, R. Perneczky, H. Jahn, M. Fiszer, J.M. Maler, R. Zimmermann, R. Bruckmoser, J. Kornhuber, P. Lewczuk, Amyloid beta peptide ratio 42/40 but not A beta 42 correlates with phospho-Tau in patients with low- and high-CSF A beta 40 load, J. Neurochem. 101 (2007) 1053-1059. https://doi.org/10.1111/j.14714159.2006.04404.x.

[25] M. Shoji, E. Matsubara, M. Kanai, M. Watanabe, T. Nakamura, Y. Tomidokoro, M. Shizuka, K. Wakabayashi, Y. Igeta, Y. Ikeda, K. Mizushima, M. Amari, K. Ishiguro, T. Kawarabayashi, Y. Harigaya, K. Okamoto, S. Hirai, Combination assay of CSF tau, A beta 140 and A beta 1-42(43) as a biochemical marker of Alzheimer's disease, J. Neurol. Sci. 158 (1998) 134-140.

[26] P. Lewczuk, H. Esselmann, M. Otto, J.M. Maler, A.W. Henkel, M.K. Henkel, O. Eikenberg, C. Antz, W.-R. Krause, U. Reulbach, J. Kornhuber, J. Wiltfang, Neurochemical diagnosis of Alzheimer's dementia by CSF Abeta42, Abeta42/Abeta40 ratio and total tau, Neurobiol. Aging. 25 (2004) 273-281. https://doi.org/10.1016/S0197-4580(03)00086-1.

[27] O. Hansson, S. Lehmann, M. Otto, H. Zetterberg, P. Lewczuk, Advantages and disadvantages of the use of the CSF Amyloid $\beta(A \beta) 42 / 40$ ratio in the diagnosis of Alzheimer's Disease, Alzheimers Res Ther. 11 (2019) 34. https://doi.org/10.1186/s13195019-0485-0.

[28] H.-H. Tsai, M. Pasi, L.-K. Tsai, Y.-F. Chen, B.-C. Lee, S.-C. Tang, P. Fotiadis, C.-Y. Huang, R.-F. Yen, J.-S. Jeng, M.E. Gurol, Microangiopathy underlying mixed-location 
intracerebral hemorrhages/microbleeds: A PiB-PET study, Neurology. 92 (2019) e774-e781. https://doi.org/10.1212/WNL.0000000000006953.

[31] K. Yaffe, A. Weston, N.R. Graff-Radford, S. Satterfield, E.M. Simonsick, S.G. Younkin, L.H. Younkin, L. Kuller, H.N. Ayonayon, J. Ding, T.B. Harris, Association of plasma beta-amyloid level and cognitive reserve with subsequent cognitive decline, JAMA. 305 (2011) 261-266. https://doi.org/10.1001/jama.2010.1995.

[32] R.J. Bateman, C. Xiong, T.L.S. Benzinger, A.M. Fagan, A. Goate, N.C. Fox, D.S. Marcus, N.J. Cairns, X. Xie, T.M. Blazey, D.M. Holtzman, A. Santacruz, V. Buckles, A. Oliver, K. Moulder, P.S. Aisen, B. Ghetti, W.E. Klunk, E. McDade, R.N. Martins, C.L. Masters, R. Mayeux, J.M. Ringman, M.N. Rossor, P.R. Schofield, R.A. Sperling, S. Salloway, J.C. Morris, Clinical and Biomarker Changes in Dominantly Inherited Alzheimer's Disease, New England Journal of Medicine. $367 \quad$ (2012) 795-804. https://doi.org/10.1056/NEJMoa1202753.

[33] Y. Yakushiji, C. Yokota, N. Yamada, Y. Kuroda, K. Minematsu, Clinical characteristics by topographical distribution of brain microbleeds, with a particular emphasis on diffuse microbleeds, J Stroke Cerebrovasc Dis. 20 (2011) 214-221. https://doi.org/10.1016/j.jstrokecerebrovasdis.2009.12.001.

[34] A. Charidimou, G. Boulouis, M.E. Gurol, C. Ayata, B.J. Bacskai, M.P. Frosch, A. Viswanathan, S.M. Greenberg, Emerging concepts in sporadic cerebral amyloid angiopathy, Brain. 140 (2017) 1829-1850. https://doi.org/10.1093/brain/awx047.

[35] J.P. Vonsattel, R.H. Myers, E.T. Hedley-Whyte, A.H. Ropper, E.D. Bird, E.P. Richardson, Cerebral amyloid angiopathy without and with cerebral hemorrhages: a comparative histological study, Ann. Neurol. 30 (1991) 637-649. https://doi.org/10.1002/ana.410300503.

[36] S.M. Greenberg, R. Al-Shahi Salman, G.J. Biessels, M. van Buchem, C. Cordonnier, J.-M. Lee, J. Montaner, J.A. Schneider, E.E. Smith, M. Vernooij, D.J. Werring, Outcome markers for clinical trials in cerebral amyloid angiopathy, Lancet Neurol. 13 (2014) 419-428. https://doi.org/10.1016/S1474-4422(14)70003-1.

[37] S. Moulin, J. Labreuche, S. Bombois, C. Rossi, G. Boulouis, H. Hénon, A. Duhamel, D. Leys, C. Cordonnier, Dementia risk after spontaneous intracerebral haemorrhage: a prospective cohort study, Lancet Neurol. 15 (2016) 820-829. https://doi.org/10.1016/S14744422(16)00130-7.

[38] A. Charidimou, G. Boulouis, L. Xiong, M. Pasi, D. Roongpiboonsopit, A. Ayres, K.M. Schwab, J. Rosand, M.E. Gurol, A. Viswanathan, S.M. Greenberg, Cortical Superficial $\begin{array}{lllll}\text { Siderosis } \quad \text { Evolution, } & \text { Stroke. } & 50 & \text { (2019) 954-962. }\end{array}$ https://doi.org/10.1161/STROKEAHA.118.023368.

[39] S. Moulin, B. Casolla, G. Kuchcinski, G. Boulouis, C. Rossi, H. Hénon, D. Leys, C. Cordonnier, Cortical superficial siderosis: A prospective observational cohort study, Neurology. 91 (2018) e132-e138. https://doi.org/10.1212/WNL.0000000000005778.

[40] Expert Panel on Detection, Evaluation, and Treatment of High Blood Cholesterol in Adults, Executive Summary of The Third Report of The National Cholesterol Education Program (NCEP) Expert Panel on Detection, Evaluation, And Treatment of High Blood Cholesterol In Adults (Adult Treatment Panel III), JAMA. 285 (2001) 2486-2497. 


\section{Tables}

Table 1. Comparison of MRI markers and demographics, clinical, CSF and genetic data between CAA, HA and mixed patients from the Magdeburg CSVD cohort.

\begin{tabular}{|c|c|c|c|c|}
\hline & $\begin{array}{c}\text { CAA } \\
n=110\end{array}$ & $\begin{array}{c}\mathrm{HA} \\
\mathrm{n}=33\end{array}$ & $\begin{array}{c}\text { Mixed } \\
\mathrm{n}=97\end{array}$ & p-value \\
\hline Age, in years & $75[8]$ & $69[13]$ & $71[11]$ & 0.01 \\
\hline Female sex, $n(\%)$ & $52(47)$ & $13(39)$ & $55(56)$ & 0.1 \\
\hline Arterial hypertension ${ }^{1}, \mathrm{n}(\%)$ & $79(88)$ & $25(86)$ & $78(98)$ & 0.03 \\
\hline Diabetes mellitus ${ }^{2}, \mathrm{n}(\%)$ & $15(18)$ & $9(33)$ & $28(39)$ & 0.005 \\
\hline BMI kg/m ${ }^{2}{ }^{3}$ & $25[4]$ & $27[4]$ & $28[4]$ & 0.001 \\
\hline $\mathrm{HDL}<1 \mathrm{mmol} / \mathrm{l}^{4}, \mathrm{n}(\%)$ & $9(13)$ & $7(28)$ & $17(30)$ & 0.02 \\
\hline ApoEع4 positivity ${ }^{5}, n(\%)$ & $10(36)$ & 0 & $8(35)$ & 0.9 \\
\hline $\operatorname{CSF} A \beta 1-40 \mathrm{pg} / \mathrm{ml}^{6}$ & $\begin{array}{l}8196(1539- \\
11932)\end{array}$ & $\begin{array}{l}7823(2440- \\
7907)\end{array}$ & $\begin{array}{l}6808(3008- \\
12994)\end{array}$ & 0.4 \\
\hline CSF A $\beta 1-42<485 p g / m l, n(\%)^{6}$ & $25(68)$ & $1(33)$ & $12(41)$ & 0.03 \\
\hline CSF A $1-42 \mathrm{pg} / \mathrm{ml}^{6}$ & $\begin{array}{l}401(169- \\
1342)\end{array}$ & $533(396-899)$ & $688(207-1269)$ & 0.04 \\
\hline CSF $A \beta$ 1-42/A $1-40$ ratio $^{6}$ & $0.6(0.3-1.4)$ & $1.1(0.7-1.6)$ & $0.9(0.3-2.8)$ & $<0.001$ \\
\hline $\mathrm{CSF}$ ttau $>350 \mathrm{pg} / \mathrm{ml}, \mathrm{n}(\%)^{6}$ & $20(54)$ & $1(33)$ & $8(28)$ & 0.03 \\
\hline CSF ttau $\mathrm{pg} / \mathrm{ml}^{6}$ & $\begin{array}{c}376(142- \\
2369)\end{array}$ & $317(276-580)$ & 279(92 -2000) & 0.3 \\
\hline CSF ptau $>70 \mathrm{pg} / \mathrm{ml}, \mathrm{n}(\%)^{6}$ & $12(32)$ & 0 & $6(21)$ & 0.3 \\
\hline CSF ptau pg/ml ${ }^{6}$ & $58(22-158)$ & $29(0-44)$ & $47(18-122)$ & 0.02 \\
\hline Lobar CMB count & $7(0-360)$ & na & $7(0-286)$ & 0.01 \\
\hline Deep CMB count & na & $0(1-7)$ & $3(1-30)$ & $<0.001$ \\
\hline Cerebellar CMB count & $0(0-5)$ & $0(0-8)$ & $0(0-16)$ & $<0.001$ \\
\hline CMB-Ratio (lobar/deep) & na & na & $2(0-72)$ & na \\
\hline Lobar ICH ( $\geq 1), n(\%)$ & $44(40)$ & na & $36(37)$ & 0.6 \\
\hline Deep ICH ( $\geq 1), n(\%)$ & na & $21(63)$ & $12(12)$ & $<0.001$ \\
\hline Cerebellar ICH ( $\geq 1), n(\%)$ & $1(1)$ & 0 & $3(3)$ & 0.3 \\
\hline cSS presence, $n(\%)$ & $56(51)$ & 2(6) & $16(17)$ & $<0.001$ \\
\hline Multiple subcortical spots, $n(\%)$ & $76(70)$ & $12(36)$ & $47(49)$ & 0.002 \\
\hline Peri-BG WMHs, $n(\%)$ & $16(15)$ & $11(33)$ & $41(43)$ & $<0.001$ \\
\hline Anterior subcortical patches, $n(\%)$ & $28(26)$ & $13(39)$ & $41(43)$ & 0.01 \\
\hline Posterior subcortical patches, $\mathrm{n}(\%)$ & $77(70)$ & $13(39)$ & $65(68)$ & 0.6 \\
\hline CSO-PVS severe $>20, n(\%)$ & $91(86)$ & $8(24)$ & $59(62)$ & $<0.001$ \\
\hline BG-PVS severe $>20, n(\%)$ & $21(20)$ & $17(52)$ & $53(55)$ & $<0.001$ \\
\hline CSO-PVS predominance, $n(\%)$ & $72(68)$ & $2(6)$ & 28(29) & $<0.001$ \\
\hline BG-PVS predominance, $n(\%)$ & $2(2)$ & $10(30)$ & 22(22) & $<0.001$ \\
\hline CSO-BG PVS equal, $n(\%)$ & $32(30)$ & $22(67)$ & $48(50)$ & 0.004 \\
\hline
\end{tabular}

Unless otherwise reported mean [SD] or median (range) is given. $A \beta, \beta$-amyloid; ApoE, Apolipoprotein E; BG, basal ganglia; BMl, body mass index; CAA, cerebral amyloid angiopathy; CMB, cerebral microbleed; CSF, cerebrospinal fluid; CSO, centrum semiovale; 
PVS, MRI-visible perivascular spaces; HA, hypertensive arteriopathy; HDL, high-density lipoprotein; $\mathrm{ICH}$, intracerebral hemorrhage; ptau, phosphorylated tau; ttau, total tau; WMHs, white matter hyperintensities; na, not applicable. P-values $\leq 0.05 / 17=2.9 \cdot 10^{-3}$ were deemed statistically significant after Bonferroni correction and marked bold. Results are adjusted for age and sex. Missing data: ${ }^{1} n=41(17 \%), \quad{ }^{2} n=59(25 \%), \quad{ }^{3} n=75(31 \%), \quad{ }^{4} n=91(38 \%)$, ${ }^{5} n=187(78 \%),{ }^{6} n=161(67 \%)$. Thresholds were set for high-density lipoprotein (HDL) at $<1 \mathrm{mmol} / \mathrm{l}[40]$, and for $A \beta 1-42$ at $485 \mathrm{pg} / \mathrm{ml}$, for ttau at $350 \mathrm{pg} / \mathrm{ml}$ [23] and for ptau at $70 \mathrm{pg} / \mathrm{ml}$ [23]. 
Table 2. Comparison of MRI markers and demographics, clinical, CSF and genetic data between mixed CAA-pattern and mixed HA-pattern patients from the Magdeburg CSVD cohort.

\begin{tabular}{|c|c|c|c|}
\hline & $\begin{array}{c}\text { Mixed CAA-pattern } \\
(\mathrm{n}=33)\end{array}$ & $\begin{array}{c}\text { Mixed HA-pattern } \\
(\mathrm{n}=64)\end{array}$ & p-value \\
\hline Age, in years & $70[12]$ & $73[9.6]$ & 0.3 \\
\hline Female sex, $n(\%)$ & $21(63)$ & $30(47)$ & 0.3 \\
\hline Arterial hypertension, $\mathrm{n}(\%)^{1}$ & $21(91)$ & $57(100)$ & 0.02 \\
\hline Diabetes mellitus, $n(\%)^{2}$ & $7(33)$ & $21(41)$ & 0.5 \\
\hline BMI kg/m $\mathrm{m}^{2,3}$ & $28[5]$ & $27[4]$ & 0.8 \\
\hline $\mathrm{HDL}<1 \mathrm{mmol} / \mathrm{l}, \mathrm{n}(\%)^{4}$ & $7(38)$ & $11(28)$ & 0.4 \\
\hline ApoE$\varepsilon 4$ positivity, $n(\%)^{5}$ & $4(57)$ & $4(25)$ & 0.07 \\
\hline CSF A $1-40 \mathrm{pg} / \mathrm{ml}^{6}$ & $7175(4118-12994)$ & $7405(3008-12641)$ & 0.9 \\
\hline CSF A $1-42<485 \mathrm{pg} / \mathrm{ml}, \mathrm{n}(\%)^{6}$ & $7(78)$ & $5(25)$ & 0.008 \\
\hline CSF A $1-42 \mathrm{pg} / \mathrm{ml}^{6}$ & $440(207-1057)$ & $704(213-1269)$ & 0.03 \\
\hline CSF $A \beta$ 1-42/A $\beta 1-40$ ratio $^{6}$ & $0.75(0.3-2.8)$ & $0.9(0.4-1.7)$ & 0.04 \\
\hline CSF ttau > $350 \mathrm{pg} / \mathrm{ml}, \mathrm{n}(\%)^{6}$ & 2(22) & $6(30)$ & 0.7 \\
\hline CSF ttau $\mathrm{pg} / \mathrm{ml}^{6}$ & $230(124-2000)$ & $290(127-1334)$ & 0.9 \\
\hline CSF ptau $>70 \mathrm{pg} / \mathrm{ml}, \mathrm{n}(\%)^{6}$ & 2(22) & $4(21)$ & 0.9 \\
\hline CSF ptau $\mathrm{pg} / \mathrm{ml}^{6}$ & $51(25-122)$ & $47(18-85)$ & 0.9 \\
\hline Lobar CMB count & $7(0-286)$ & $4(1-150)$ & 0.03 \\
\hline Deep CMB count & $2(1-18)$ & $3(1-30)$ & 0.05 \\
\hline Cerebellar CMB count & $0(0-14)$ & $1(0-16)$ & 0.9 \\
\hline CMB-Ratio (lobar/deep) & $3(0-72)$ & $1.2(0-50)$ & $<0.001$ \\
\hline Lobar ICH ( $\geq 1), n(\%)$ & $19(58)$ & $17(27)$ & 0.03 \\
\hline Deep ICH $(\geq 1), n(\%)$ & 1(3) & $11(17)$ & 0.05 \\
\hline Cerebellar ICH ( $\geq 1), n(\%)$ & 0 & $3(5)$ & 0.2 \\
\hline cSS presence, $n(\%)$ & $16(49)$ & na & na \\
\hline Multiple subcortical spots, $n(\%)$ & $16(50)$ & $31(49)$ & 0.9 \\
\hline Peri-BG WMHs, $n(\%)$ & $4(13)$ & $37(59)$ & $<0.001$ \\
\hline Anterior subcortical patches, $n(\%)$ & $11(34)$ & $30(48)$ & 0.2 \\
\hline Posterior subcortical patches, $n(\%)$ & $21(66)$ & $44(70)$ & 0.7 \\
\hline CSO-PVS severe > $20, n(\%)$ & $30(91)$ & $29(46)$ & $<0.001$ \\
\hline BG-PVS severe $>20, n(\%)$ & $5(15)$ & $48(76)$ & $<0.001$ \\
\hline CSO-PVS predominance, $\mathrm{n}(\%)$ & $27(82)$ & na & na \\
\hline BG-PVS predominance, $n(\%)$ & $2(6)$ & $19(30)$ & 0.007 \\
\hline CSO- \& BG-PVS equal, $n(\%)$ & $4(12)$ & $44(70)$ & $<0.001$ \\
\hline
\end{tabular}

Unless otherwise reported mean [SD] or median (range) is given. $A \beta, \beta$-amyloid; $A p o E$, Apolipoprotein E; BG, basal ganglia; BMI, body mass index; CAA, cerebral amyloid angiopathy; CMB, cerebral microbleed; CSF, cerebrospinal fluid; CSO, centrum semiovale; PVS, MRI-visible perivascular spaces; HA, hypertensive arteriopathy; HDL, high-density lipoprotein; $\mathrm{ICH}$, intracerebral hemorrhage; ptau, phosphorylated tau; ttau, total tau; WMHs, white matter hyperintensities; na, not applicable. P-values $\leq 0.05 / 17=2.9 \cdot 10^{-3}$ were deemed statistically significant after Bonferroni correction and marked bold. Results are adjusted for age and sex. Missing data: ${ }^{1} n=17(18 \%),{ }^{2} n=25(24 \%),{ }^{3} n=35(34 \%),{ }^{4} n=41(42 \%),{ }^{5} n=74(76 \%)$, ${ }^{6} \mathrm{n}=58(56 \%)$. Thresholds were set for high-density lipoprotein (HDL) at $<1 \mathrm{mmol} / /$ [40], and for $\mathrm{A} \beta 1-42$ at $485 \mathrm{pg} / \mathrm{ml}$, for ttau at 350pg/ml [23] and for ptau at $70 \mathrm{pg} / \mathrm{ml}$ [23]. 


\section{Figure legend}

Figure 1: Mixed CAA- and mixed HA pattern.

Figure A-D demonstrates the $1.5 \mathrm{~T}$ MRI of a 77 -year old man with mixed CAApattern. The T2-weighted images show in A frequent MRI-visible perivascular spaces (PVS) in the centrum semiovale (CSO) (inlay) and in B mild PVS in the basal ganglia (BG) (inlay), indicative of a CSO-PVS predominance pattern. The T2*-gradientrecalled echo (GRE) image in $C$ exhibits a disseminated form of cortical superficial siderosis (arrow) and in D one deep cerebral microbleed (arrow) in the left thalamus.

Figure E-G demonstrates the 1.5 T MRI of a 69-year old man with mixed HA-pattern. The T2-weighted images show in E mild MRI-visible PVS in the CSO (inlay) and in F severe PVS in the BG (inlay), indicative of a BG-PVS predominance pattern. The T2*-GRE image in G exhibits three lobar CMBs in the right frontal and left parietal cortex (arrowheads) and in $\mathrm{H}$ two deep CMBs in the left and right thalamus (arrowheads). Note in $\mathrm{H}$ another lobar $\mathrm{CMB}$ in the right temporal cortex (arrowheads). 
A T2 image

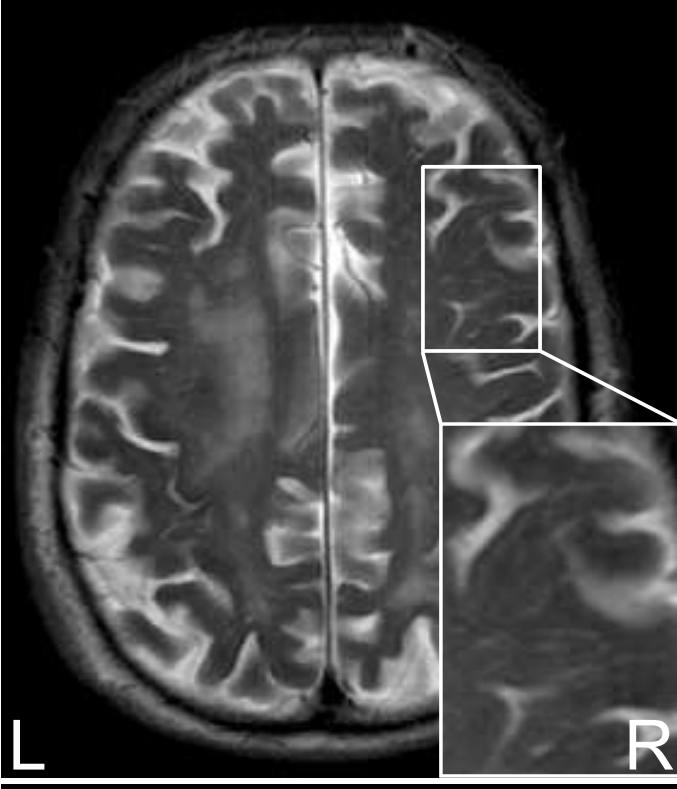

C T2*-GRE image

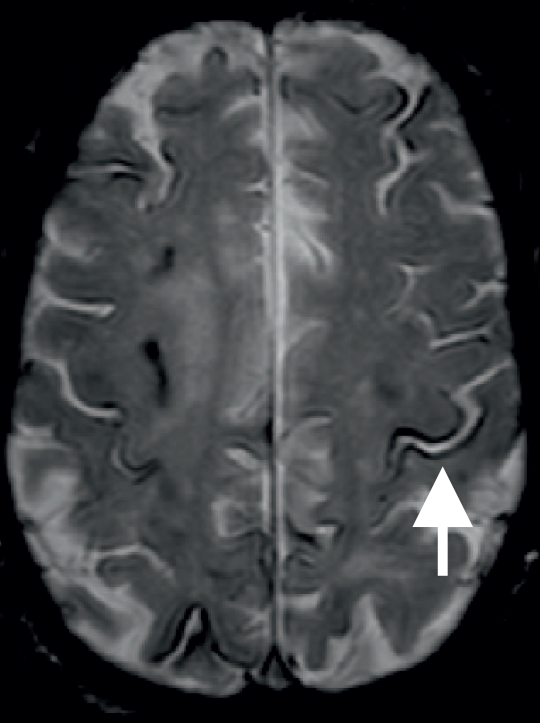

B T2 image

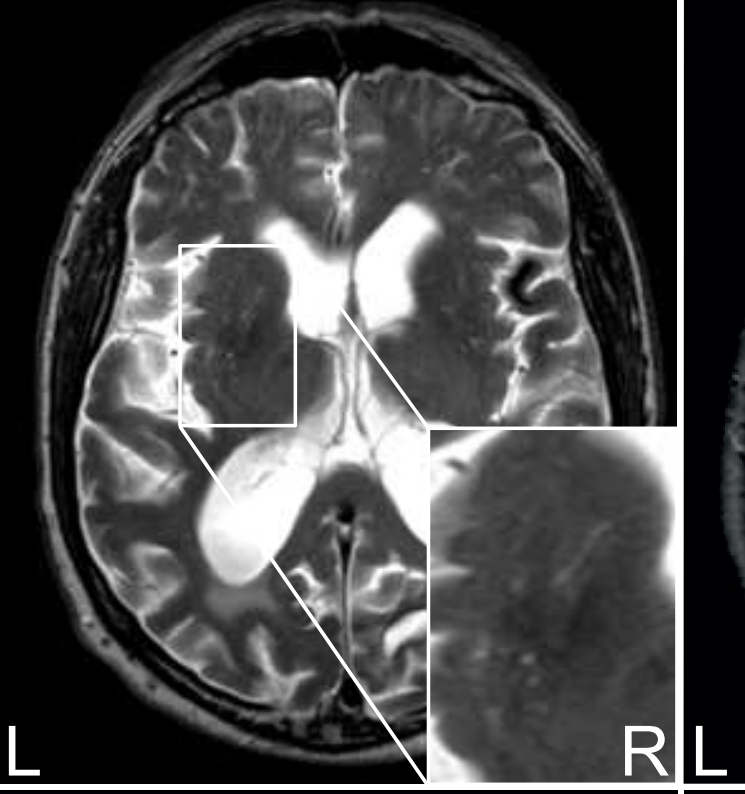

D T2*-GRE image

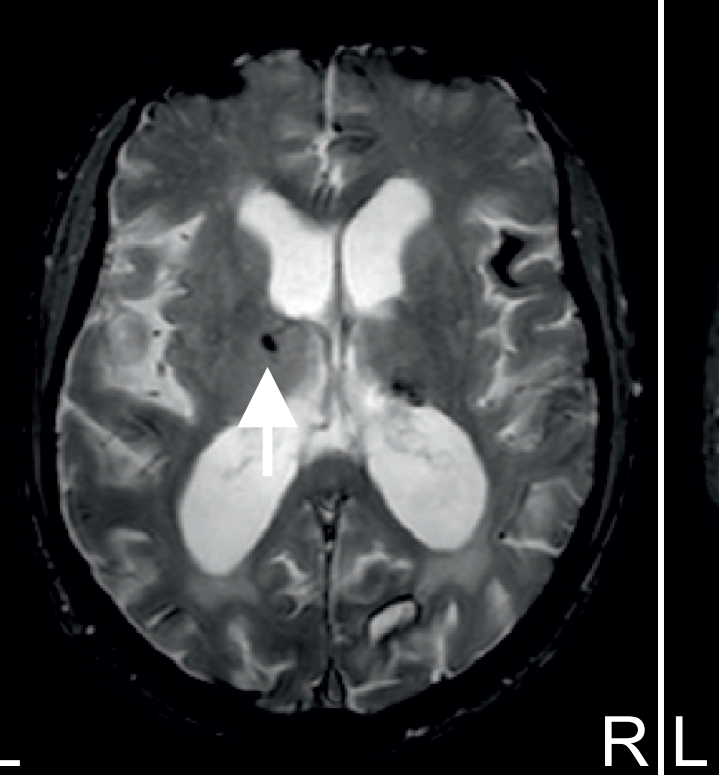

E T2 image

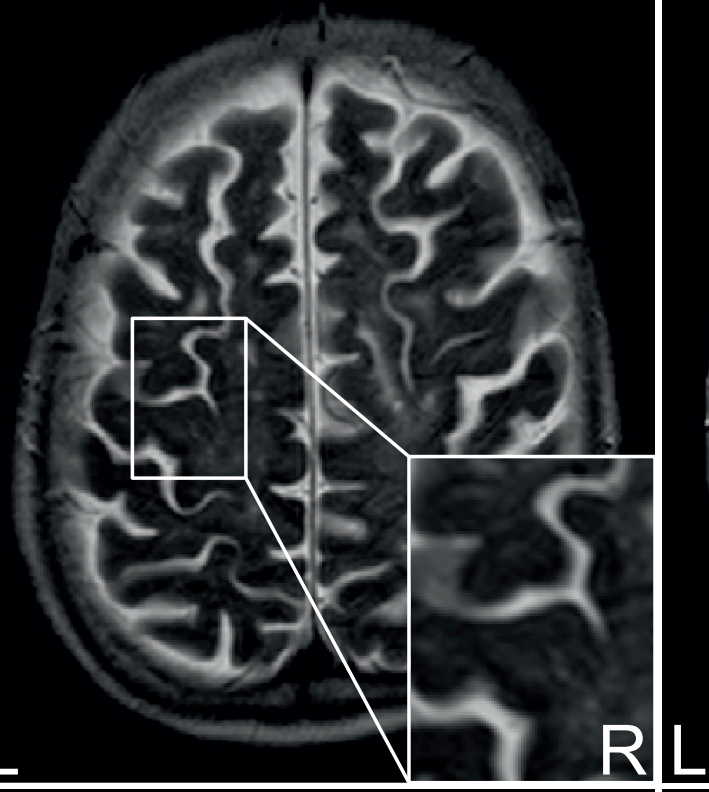

G T2*-GRE image
F T2 image

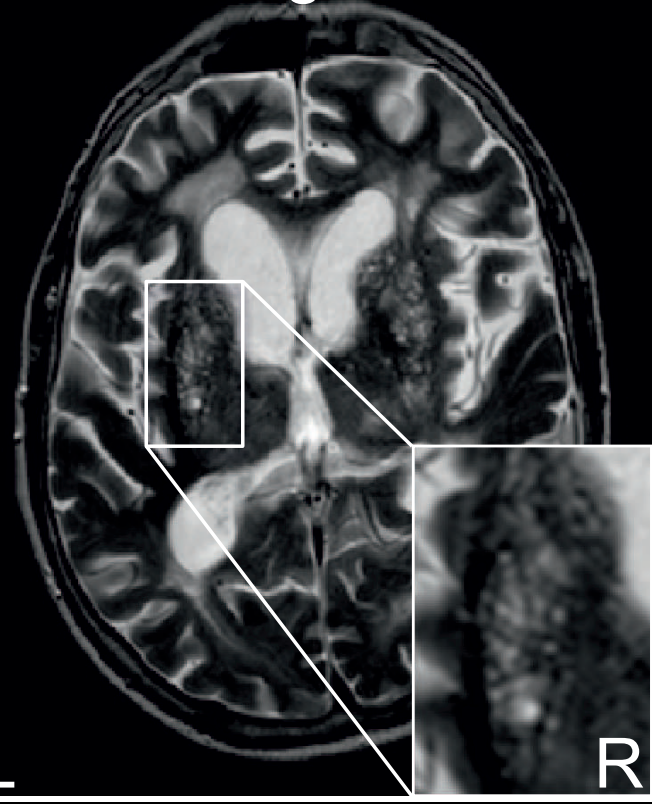

H T2*-GRE image

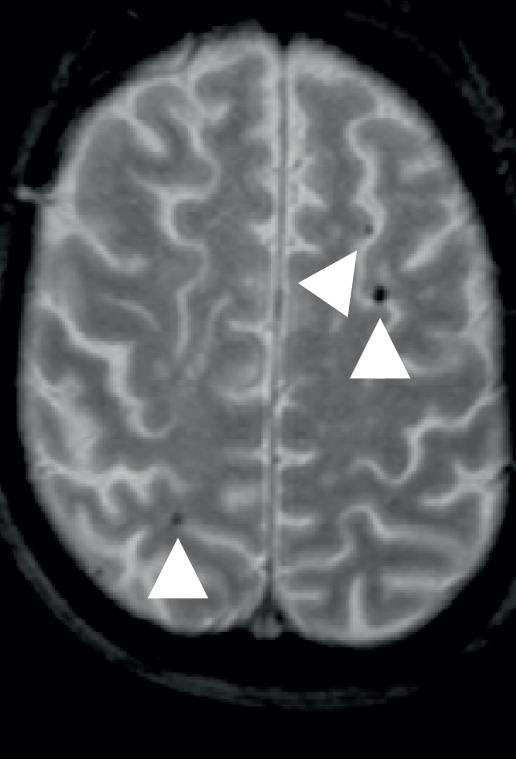

RL

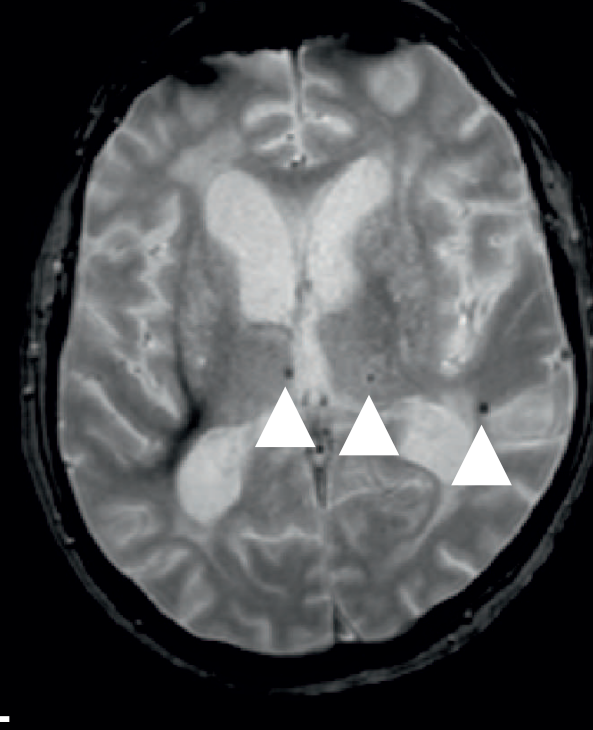

R 